Asian Journal of Information Technology 11 (1): 14-21, 2012

ISSN: $1682-3915$

(C) Medwell Journals, 2012

\title{
Design and Implementation of Blocks-Based Educational Courseware for Children with Learning Disabilities
}

\author{
Afiza Ismail, Nazlia Omar and Abdullah Mohd Zin \\ Center for Software Technology and Management, \\ Faculty of Information Science and Technology, \\ Universiti Kebangsaan Malaysia, 43600 Bangi, Selangor, Malaysia
}

\begin{abstract}
Customized education is now being seriously considered for children with learning disability such as autism who have serious impairments with social, emotional and communication skills. One of the approaches for providing this type of education is by using highly personalized educational courseware. The aim of this study is to describe the design and implementation of blocks for developing educational courseware for autistic children with learning disability. The availability of these blocks enables end-users (such as parents or teachers) to build personalized educational courseware within the block-based software development environment. The process of designing and implementing programming blocks involves four main activities: blocks identification process, blocks design, blocks implementation and evaluation. In order to prepare the autistic child to learn to read, four blocks that are required in the pre-reading stage: apart from these three blocks, there are three types of blocks that need to be provided so that application programs can be properly developed. The suitability of the blocks have been shown by using simple case studies.
\end{abstract}

Key words: Block-based software development, component-based software development, end-user programming, autism, disability, Malaysia

\section{INTRODUCTION}

Customized education is an education system in which technologies and organizational skills are combined to provide for the individual's educational needs (Sokolov, 2001). In an environment where students differ greatly in their abilities, a uniform education cannot be uniformly challenging for all students. Reigeluth (1997) states that we need customization to replace standardization in order to have an education system that is focused on learning (attaining high standards) rather than on sorting.

Special education is one of the areas where customized education will be useful. The main task of special education is to educate children with learning disabilities. The term learning disability describes a broad category of developmental disorder and refers to a deficit in learning in one or more domains which can include reading, mathematics and writing (Lyon et al., 2003). It includes all types of disabilities that affect cognition such as dyslexia, dysgraphia, dyscalculia, attention deficit disorder, autism and down syndrome (Bohman and
Anderson, 2005). Children with learning disabilities are as smart as or smarter than their peers if they are given the right support and intervention. Parents can help by encouraging their strengths, knowing their weaknesses and use the right learning strategies for dealing with specific difficulties.

This study addresses a specific group of children with learning disabilities that is children with autism. This group of children have substantial difficulties in social interaction and communication and the presence of unusual behaviors and interests. They have serious impairments with social, emotional and communication skills and they might repeat certain behaviors again and again. Most of them also have different ways of learning, paying attention or reacting to things.

Computer-based learning is currently being utilized as a means of enabling new ways of learning and teaching in children with autism (Powell, 1996). A study by Williams et al. (2002) indicates that 3-5 years old children with autism spent more time on reading material when they accessed it through a computer. The study also, found that these children were less resistant to the use of

Corresponding Author: Afiza Ismail, Center for Software Technology and Management, Faculty of Information Science and Technology, Universiti Kebangsaan Malaysia, 43600 Bangi, Selangor, Malaysia 
this technology in learning. Moore and Calvert (2000) found that computer software can create an interesting and simulating environment for children with autism. Since, each autistic child has different ways of learning, they should be taught in a customized education environment. This implies that each one of them should be given the opportunity to use the educational software that is specifically customized for him/her. However, most of the educational softwares currently available in the market do not provide provision for customization.

The objective of this study is to describe a research that was carried out in order to develop customizable and tailorable softwares for children with autism. This research is based on the block-based software development approach which is a new end-user programming approach. The description of this software development approach is given by Zin (2011). In a Block-Based Software Development Environment, many blocks will be made available for a problem domain. A block is a single layer software component that can be used to perform a specific task. A block consists of four main elements: attributes, behaviour, GUI elements and interfacing with other blocks. A block can be customized by changing the values of the attributes. Blocks are designed and implemented by the block developers who are professional programmers. End-users such as parents, teachers, managers, administrators, engineers and scientists will develop an application by selecting and integrating these blocks. The process of integrating blocks can be done by using a block integrating tool, a software tool that can be used by end-users to integrate blocks (Sarif et al., 2011).

\section{MATERIALS AND METHODS}

In the previous study (Ismail et al., 2009), researchers have outlined skills that are required by children with autism. These skills can be divided into four categories as follows:

- Gross motor skills refer to the activities related to movement and mobility

- Fine motor skills (art and craft) refer to activities such as pre-drawing skills, learning to draw and learning to colour a picture

- Language development skills refers to a child's ability to vocalize or speak words and sentences and their ability to understand what is said to them by other people

- Reading, writing and early numbers skills are considered to be very important skills because once mastered they open the doors to greater independence in the society
The work focus on the last skill, specifically on reading. We focus on this particular aspect since the use of computers in developing reading skills have been recognized by a number of researchers. According to Williams et al. (2002), computer-based instruction was effective in teaching children with autism to read. Murray (1997) suggests that the exposure to texts and the potential to survey them at the child's leisure using technology, such as computers, can motivate a child with ASD to develop reading skills. Heimann et al. (1995) noted that the intervention of motivating multimedia as well as planning and monitoring by teachers, parents and clinicians will increase both reading and phonological awareness in children in ASD.

In order to enable end-user programmers to develop applications to help autistic children to learn how to read, some programming blocks need to be provided. The process of providing programming blocks involves four main activities: blocks identification process, blocks design, blocks implementation and evaluation.

Block identification is the process of identifying all blocks that need to be provided for end-user developers to develop application in a particular domain. The process of identifying blocks is done by carrying out the domain analysis. In the case of early reading skills for autistic children, we can see that there are three types of skills that are required: pre-reading, learning to read and advanced reading. In this study, we describe the blocks for pre-reading. Pre reading skills are the skills needed by children to enable them become a reader. Many of these skills are learnt naturally during the course of a normal childhood, at home and in the nursery/preschool environment (The Family Learning, 2011).

Block design starts with the GUI design. During this design process, elements of the blocks such as labels, texts buttons, audio and video controllers are identified and positioned on the block's layout. To enable blocks to be linked with other blocks, every block has three standard navigation buttons: previous, next and exit.

Block are implemented by using JavaBeans concept and methodology. According to O'Neil and Schildt (1998), JavaBeans is the software component architecture for Java. It is an exciting and important new technology that allows programmers to efficiently construct applications by configuring and connecting components called Beans. A Bean is a reusable software component written in Java. That standard defines the mechanism by which components perform common tasks such as communicating with each other interacting with tools and saving/restoring their configuration. Properties, events and methods are fundamental to understanding and using components. A property is a subset of a component's 
state. A programmer may change the behaviour of that component by modifying its properties. An event is a notification that is generated by a component when there is some change in its state. Methods can be invoked to execute code in a component.

In the current implementation, a block is packaged as a JAR (Java Archive) file. JAR files provide a standard mechanism to compress and package a set of files for distribution to users. The following files may be packaged into one JAR file:

- The class files

- The attribute file

- Any supporting class files

- Static images

- Audio and video clips

- Configuration data

- Any other files required for the software to operate

\section{RESULTS AND DISCUSSION}

In order to prepare an autistic child to learn to read, four blocks that are required in the pre-reading stage:

- The block for learning of alphabets where the child will learn about the alphabets or letters

- The block for learning of two syllables where the child will learn on how to combine the alphabets to construct two syllables

- The block for learning of three syllables where the child will learn on how to combine the alphabets to construct three syllables

- The block for learning of words where the child will use two syllables to construct word (such as noun, verbs and idioms)

Since, image and sound play vital role in educating the autistic child to read (Grandin, 1995) these three elements must be included in blocks' attributes and endusers should be able to customize the blocks by changing these attributes to suit the child's preferences and needs. Other attributes of these blocks are text, title of the block and colour. Application developers such as parents or teachers are also allowed to edit certain attributes of the blocks such as the colour of the background, the colour of the letter and also to adjust on the volume speaker (such as mute or decrease/increase volume).

Learning of alphabets block: The design layout for block is shown in Fig. 1. This block consists of 7 attributes:

- Name of the instance of the block

- Letter

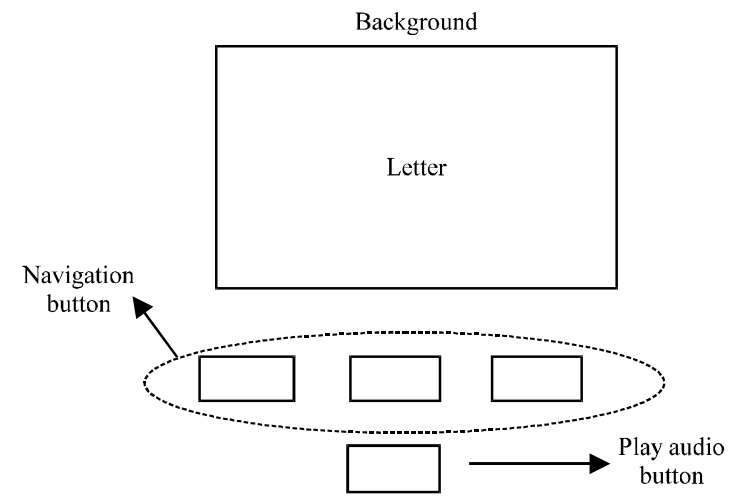

Fig. 1: The design layout of the learning of alphabets block

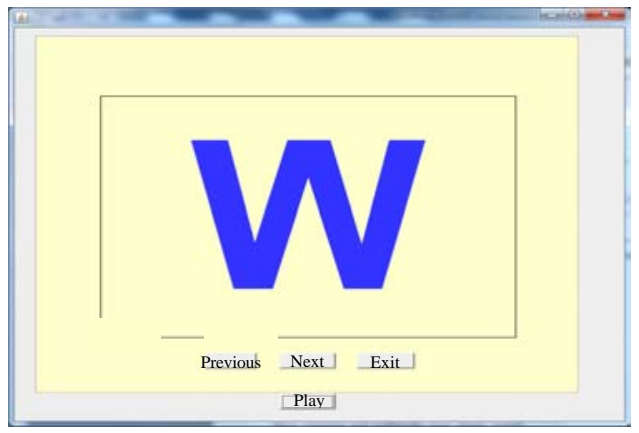

Fig. 2: An instance of the learning of alphabets block

- Text colour

- Text size

- Text font

- Background colour

- Audio file name

End-user developers can change these attributes in order to create an instance of the block. For example, an instance of block for learning of alphabet is shown in Fig. 2. In this case the letter is assigned as W.

Learning of syllables blocks: The design layouts for these blocks are shown in Fig. 3 and 4. The block in Fig. 3 consists of 8 attributes:

- Name of the instance of the block

- Syllable1

- Syllable2

- Text colour

- Text size

- Text font

- Background colour

- Audio file name

while an additional attribute for Syllable 3 is required for the block in Fig. 4. 


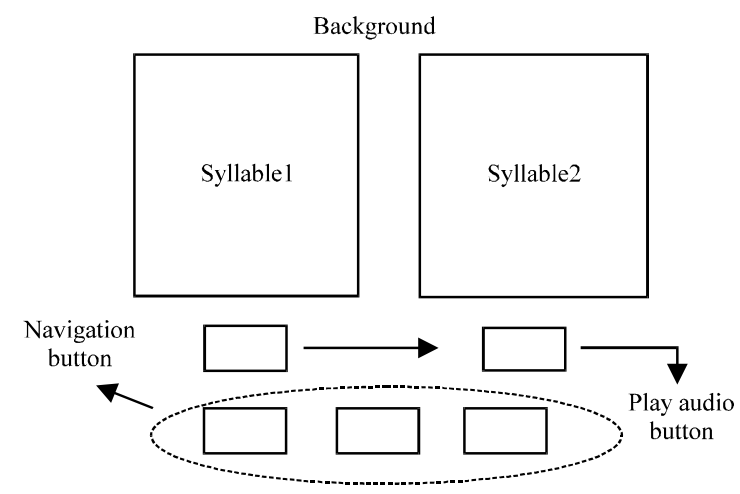

Fig. 3: The design layout of the learning of two syllables block

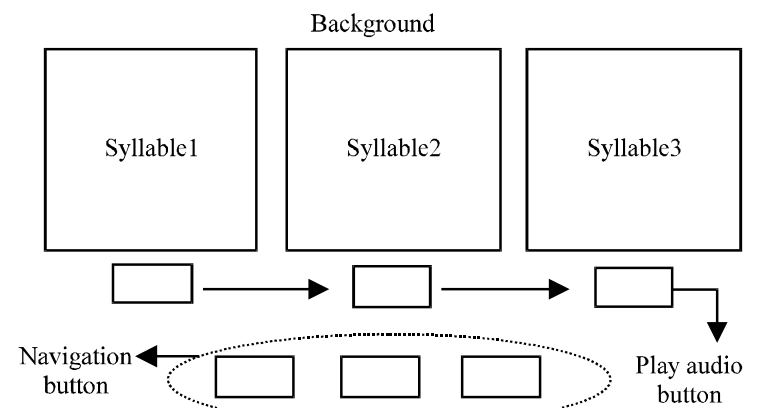

Fig. 4: The design layout of the learning of three syllables block

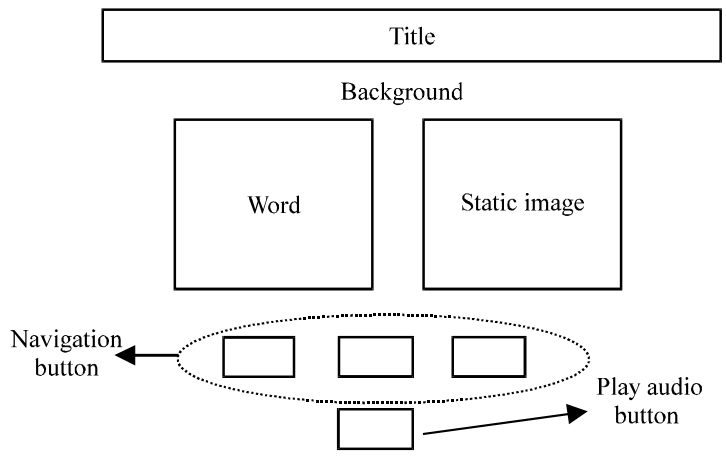

Fig. 5: The design layout of the learning of words block

Learning of words block: The design layout for this block is shown in Fig. 5. This block consists of 9 attributes: name of the instance of the block, title of the block, word, image, text colour, text size, text font, background colour and audio file name. An instance of learning of words block is shown in Fig. 6.

Supporting blocks: Apart from these three blocks, there are three types of blocks that need to be provided so that application programs can be properly developed. These blocks are:

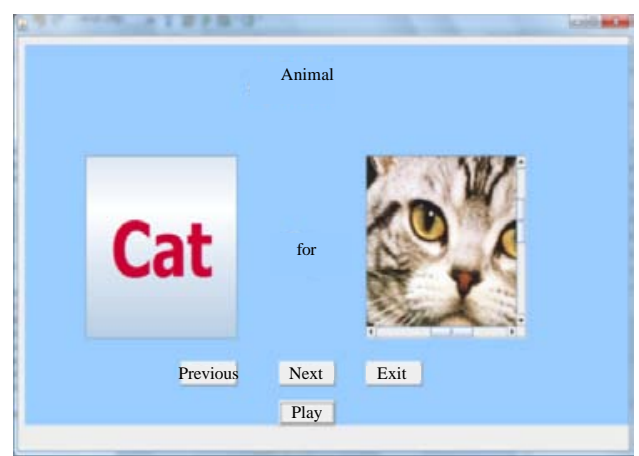

Fig. 6: An instance of the learning of words block

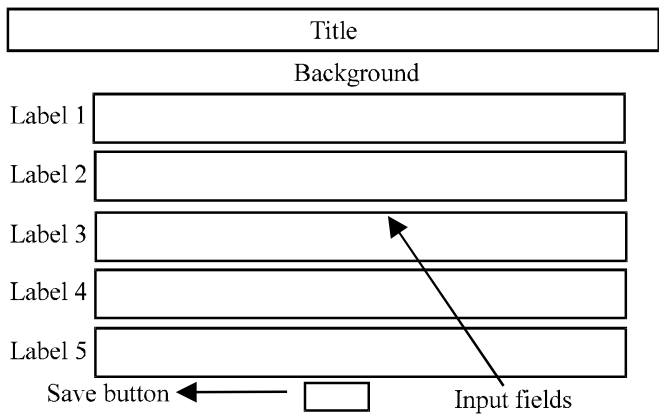

Fig. 7: The design layout of the candidate profile block

Candidate profile block: This block can be used for storing information regarding the autistic child such as name, identifier of the child's group, child's preferences and sensitivity. That information will be used later as input parameter in another block. For example if the parents or the teachers input the child's name as Tom, the value of the parameter will be passed to another block that will receive it as the input parameter. The next block will display Tom as the name of the child.

Welcoming block: The block contains text welcoming the autistic child in the learning session.

Exercise block: Activity/exercise block and/or gaming block can be incorporated into an application in order to test on the child's ability in understanding the skills that they have learned. There are three possible blocks for this purpose. The three exercise blocks focus on learning letters, syllables and words, respectively.

Candidate profile block: The design layout of the block is shown in Fig. 7. Since, this block is supposed to be the first block to be used, it is equipped with only one save button. This block consists of 8 attributes: Name of the instance of the block, Title of the block, input fieldl, input field2, input field3, input field4, input field5 and background colour. An instance of the candidate profile block is shown in Fig. 8. 


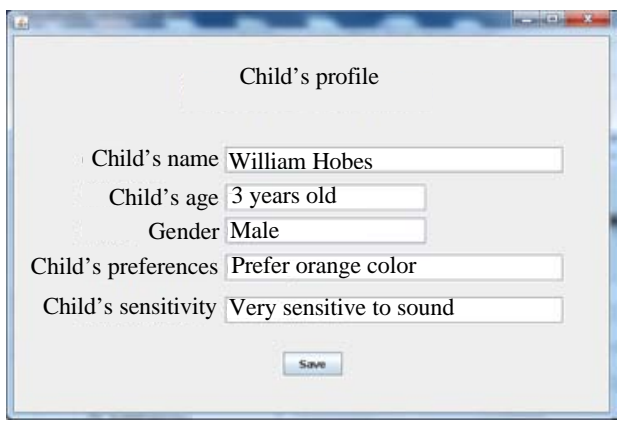

Fig. 8: An instance of the candidate profile block

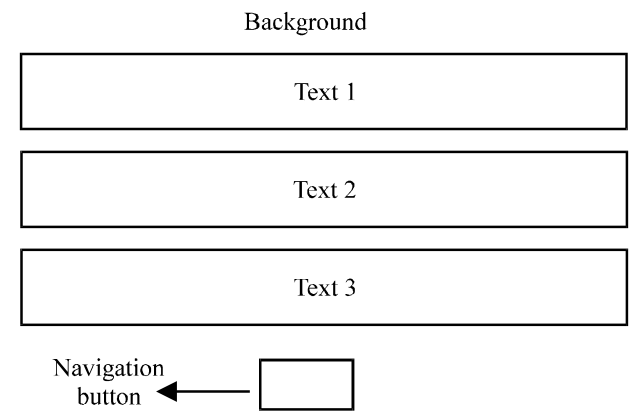

Fig. 9: The design layout of the welcoming block

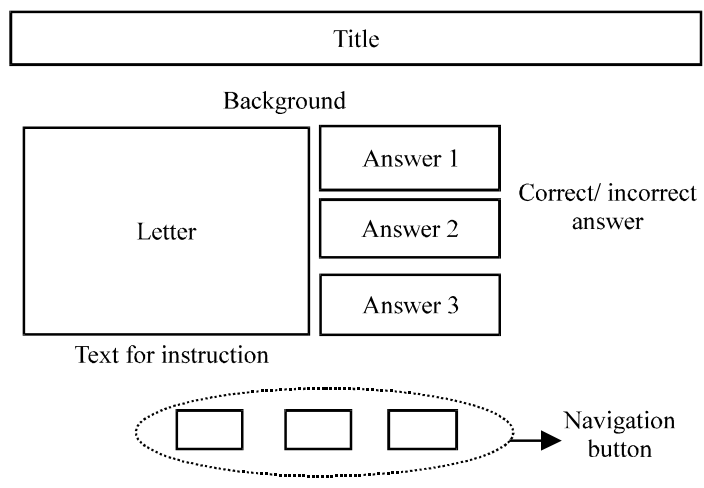

Fig. 10: The design layout of the letter exercise block

Welcoming block: The design layout of the block is shown in Fig. 9. The parents or teachers can input the text welcoming the autistic student in the learning session. This block consists of 8 attributes: name of the instance of the block, Text1, Text2, Text3, Text colour, Text size, Text font and background colour.

Activity/exercise block: The layout design for the three activity/exercise blocks are shown in Fig. 10-12. Figure 10 shows a block for testing the children's knowledge of letters. Parents or teachers can edit the text of the letter/alphabet as well as the audio file. They can also enter three choices of answers related to the letter given

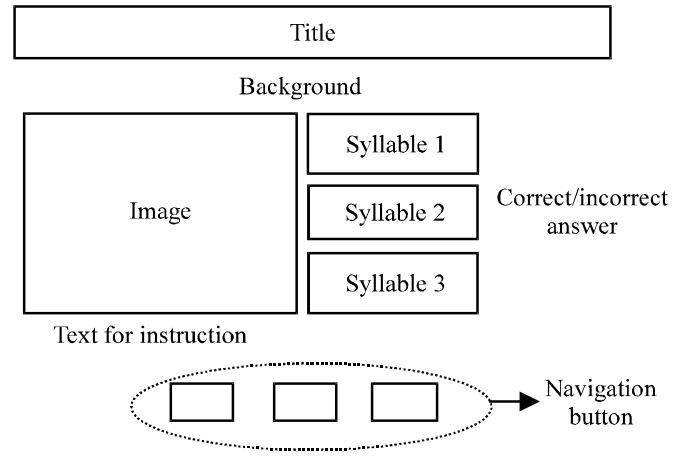

Fig. 11: The design layout of the syllable exercise block

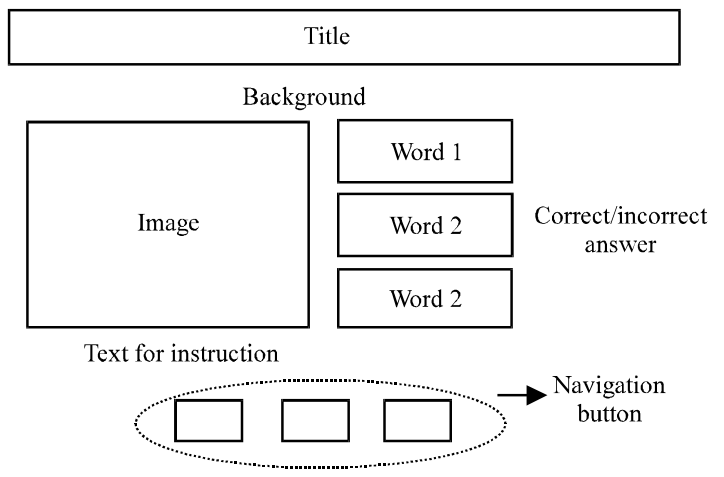

Fig. 12: The design layout of the word exercise block

and can also display/output the answer as correct or incorrect. This block consists of 15 attributes: name of the instance of the block, title of the block, text for instruction, letter, 3 attributes for answers, 3 attributes for correct answers, text colour, text size, text font, background colour and audio file name.

Figure 11 shows a block for testing children's understanding of syllables. Parents or teachers can edit the image as well as the audio file. They can also enter three choices of answers related to the image given and can also display/output the answer as correct or incorrect. This block also consists of 15 attributes.

Figure 12 shows the design layout of a block for word exercise. Parents or teachers can edit the image of the noun as well as the audio file. They can also enter three choices of answers related to the image given and can also input the answer as correct or incorrect. This block also consists of 15 attributes.

A case study: There are a number of applications that can be developed for teaching autistic children to read by using the blocks that have been implemented. In order to develop an application, an end-user programmer needs to use the block integration tool in order to: 

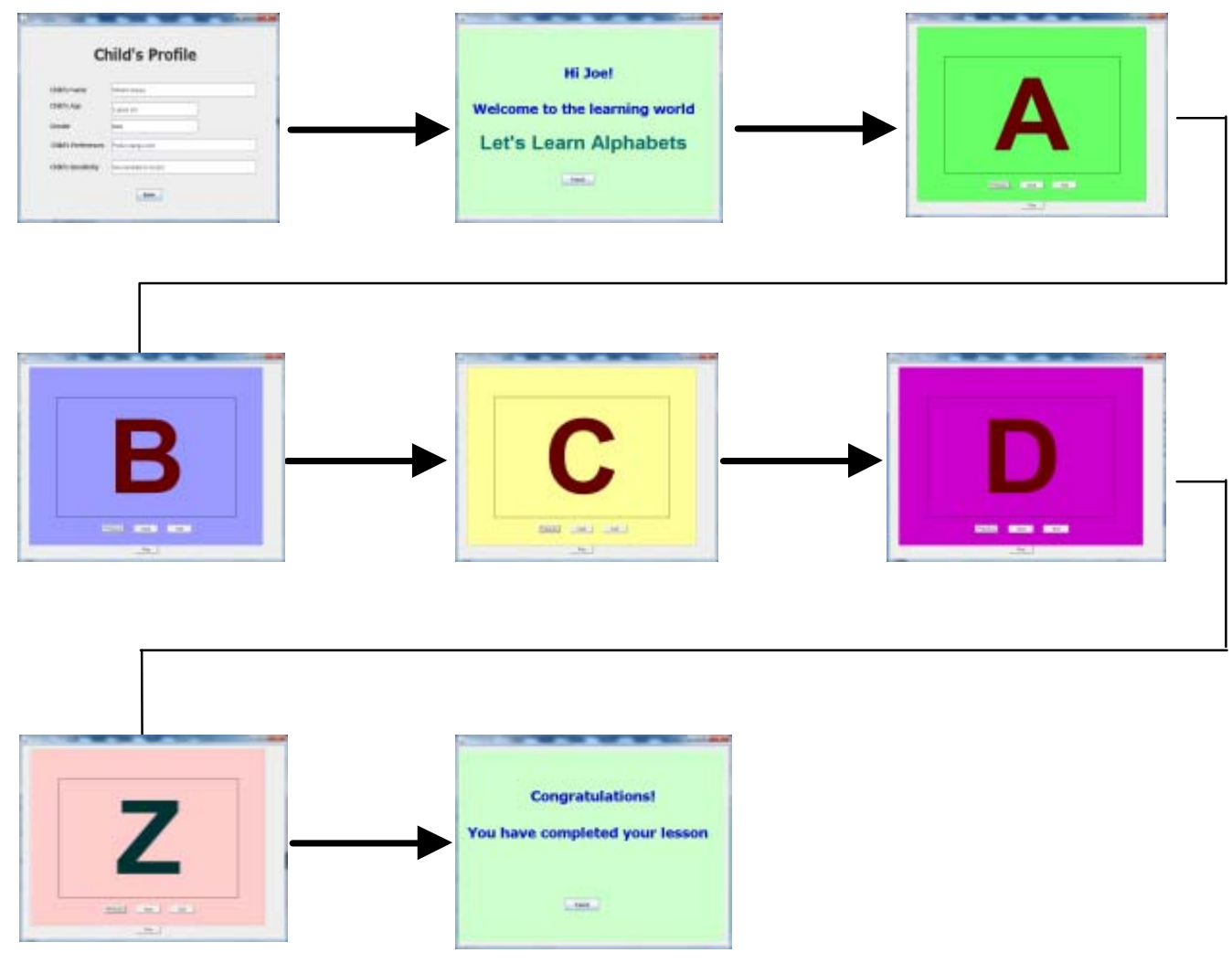

Fig. 13: The blocks are linked together (sequentially) to develop an application related to learning letters

- Select blocks

- Create instance of the blocks

- Link the blocks with other blocks

- Compile the application

- Run the application

There are three ways for linking one block with another block: sequential link, deterministic selection and non-deterministic selection. If Block-A is sequentially linked to Block-B then after Block-A has been executed, Block-B will be executed. In deterministic selection, after Block-A is executed, Block-Bn will be executed based on the condition. In non-deterministic selection, after Block$\mathrm{A}$ is executed, one of Block-Bn will be selected at random.

Getting to know letter application: This is a simple application to teach a child to know letters. Three blocks can be used: candidate profile block, welcoming block and learning of alphabet block.

Each of these blocks needs to be customized to create instances of the blocks. The customization is done by changing values of certain attributes of the block to suit the needs of the autistic child. By doing this, the application can be specifically developed for a particular autistic child.
In the case of CandidateProfile block, only one instance is created. For the welcoming block, two instances are created: one for welcoming the child and the other for congratulating him once he has completed the lesson. For the third block, twenty six (A-Z) instances need to be created. All of the instances of these blocks can then be linked by using sequential link as shown in Fig. 13.

Getting to know syllables: This is an application to teach a child to know the syllables such as RO, BOT and TI, GER. These are known as two syllables. The syllables such as AL, PHA, BET and TRI, AN and GLE are known as three syllables. Four blocks can be used: candidate profile block, welcoming block, learning of two syllables block and learning of three syllables block.

Each of these blocks needs to be customized to create instances of the blocks. In the case of welcoming block and candidate profile block, only one instance is created for each one of them. For the third block, two instances need to be created and for the fifth block, two instances need to be created. All of the instances of these blocks can then be linked by using sequential link. 


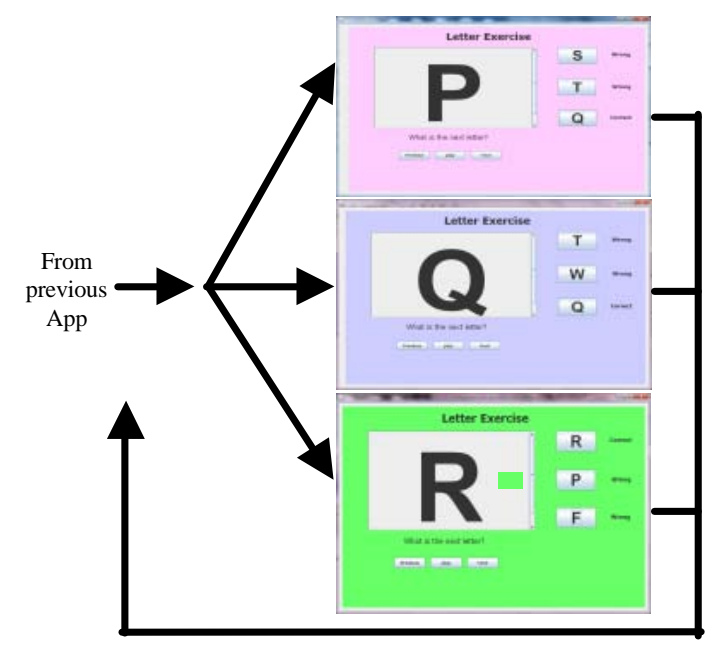

Fig. 14: The blocks are linked together (nondeterministic) to develop an application related to learning letters

Getting to know words: This is an application to teach a child to know the words CAR, APPLE and HAT. Three blocks can be used: Candidate profile block, welcoming block and learning of word block.

Each of these blocks needs to be customized to create instances of the blocks. In the case of welcoming block and candidate profile block, only one instance is created for each one of them. For the third block, three instances need to be created. All of the instances of these blocks can then be linked by using sequential link.

Advanced getting to know letter application: A more advanced application can be created by including an exercise at the end of the lesson. For this purpose the letter exercise block need to be used. Three instances of the block can be created. To make it more interesting, these blocks can be linked together by using nondeterministic link as shown in Fig. 14. In this case, one of the options is selected at random.

\section{CONCLUSION}

Block-based software development approach offers tailorable design architectures that enables end users to developed customized applications to suit different needs of users. This study describes the design and implementation of blocks for pre-reading that can assist end users such as teachers and parents in their effort to educate children with learning disabilities, particularly autism. The availability of these blocks enables the development of educational courseware that will cater each child needs individually, easily and effectively. The customized education environment provided can accelerate the child's learning and thus able to achieve the learning objective in a shorter period of time. The suitability of the blocks for developing applications have been shown by using them in the development of some simple applications.

A further study is currently being carried out to identify, design and implement all the blocks required for developing applications for autistic children with learning disability. By having a complete set of blocks, complex applications for these chidren can be developed.

\section{REFERENCES}

Bohman, P.R. and S. Anderson, 2005. A conceptual framework for accessibility tools to benefit users with cognitive disabilities. Proceedings of the International Cross-Disciplinary Workshop on Web Accessibility, May 18, 2005, ACM, New York, USA., pp: 85-89.

Grandin, T., 1995. How People with Autism Think. In: Learning and Cognition in Autism, Schopler, E. and G.B. Mesibov (Eds.). Plenum, New York, USA., ISBN-13:9780471241867, pp: 137-156.

Heimann, M., K.E. Nelson, T. Tjus and C. Gillberg, 1995. Increasing reading and communication skills in children with autism through an interactive multimedia computer program. J. Autism Develop. Disorder, 25: 459-480.

Ismail, A., N. Omar and A.M. Zin, 2009. Developing learning software for children with learning disabilities through block-based development approach. Proceedings of the International Conference on Electrical Engineering and Informatics, August 5-7, 2009, Selangor, Malaysia.

Lyon, G.R., J.M. Fletcher and M.C. Barnes, 2003. Learning Disabilities. In: Child Psychopathology, 2nd Edn., Mash, E.J. and R.A. Barkley (Eds.). Guilford Press, New York, USA., pp: 520-588.

Moore, M. and S. Calvert, 2000. Brief report: Vocabulary acquisition for children with autism: Teacher or computer instruction. J. Autism Dev. Disord., 30: $359-362$.

Murray, D.K.C., 1997. Autism and Information Technology: Therapy with Computers. Autism and Learning. In: A Guide to Good Practice, Powell, S. and R. Jordan (Eds.). David Fulton Publishers, London, UK.

O'Neil, J. and H. Schildt, 1998. JavaBeans TM Programming from the Ground Up. Osborne/McGrawHill, California, USA., ISBN-13:9780078824777, Pages: 487. 
Powell, S., 1996. The use of computers in teaching people with autism. Proceedings of the National Autistic Society Conference, July 10-13, 1996, London, UK., pp: $128-132$.

Reigeluth, C.M., 1997. Educational standards: To standardize or to customize learning?. Phi Delta Kappan, 79: 202-206.

Sarif, S.N., S. Idris and A.M. Zin, 2011. The design of blocks integration tool to support end-user programming. Proceedings of the International Conference on Electrical Engineering and Informatics, July 17-19, 2011, Bandung, Indonesia.
Sokolov, M., 2001. Technology's impact on society: The issue of mass-customized education. Technol. Forecast. Soc. Change, 68: 195-206.

The Family Learning, 2011. Pre-reading skills. UK., http://www.familylearning.org.uk/pre-reading_skills. html.

Williams, C., B. Wright, G. Callaghan and B. Coughlan, 2002. Do children with autism learn to read more readily by computer assisted instruction or traditional book methods. Autism, 6: 71-91.

Zin, A.M., 2011. Block-based approach for end-user software development. Asian J. Inform. Technol., 10: $249-258$. 